\title{
Options for Dealing with Pressure Dependence of Pulse Wave Velocity as a Measure of Arterial Stiffness: An Update of Cardio-Ankle Vascular Index (CAVI) and CAVI
}

\author{
Bart Spronck $^{\mathrm{a}, \mathrm{b}} \quad$ Tammo Delhaas $^{\mathrm{b}}$ Mark Butlin $^{\mathrm{a}} \quad$ Koen D. Reesink $^{\mathrm{b}}$ \\ Alberto P. Avolio ${ }^{a}$ \\ a Department of Biomedical Sciences, Faculty of Medicine and Health Sciences, Macquarie \\ University, Sydney, NSW, Australia; ${ }^{b}$ Department of Biomedical Engineering, CARIM School \\ for Cardiovascular Diseases, Maastricht University, Maastricht, The Netherlands
}

\section{Keywords}

Arteriosclerosis · Blood pressure correction · Arterial compliance $\cdot$ Hypertension

\begin{abstract}
Pulse wave velocity (PWV), a marker of arterial stiffness, is known to change instantaneously with changes in blood pressure. In this mini-review, we discuss two main approaches for handling the blood pressure dependence of PWV: (1) converting PWV into a pressure-independent index, and (2) correcting PWV per se for the pressure dependence. Under option 1, we focus on cardio-ankle vascular index (CAVI). CAVI is essentially a form of stiffness index $\beta-$ CAVI is estimated for a (heart-to-ankle) trajectory, whereas $\beta$ is estimated for a single artery from pressure and diameter measurements. Stiffness index $\beta$, and therefore also CAVI, have been shown to theoretically exhibit a slight residual blood pressure dependence due to the use of diastolic blood pressure instead of a fixed reference blood pressure. Additionally, CAVI exhibits pressure dependence due to the use of an estimated derivative of the pressure-diameter relationship. In this mini-review, we will address CAVI's blood pressure dependence theoretically, but also statistically. Furthermore, we review corrected indices (CAVI $I_{0}$ and $\left.\beta_{0}\right)$ that theoretically do not show a residual blood pressure dependence. Under option 2, three ways of correcting PWV are reviewed: (1) using an exponential relationship between pressure and cross-sectional area, (2) by statistical model adjustment, and (3) through reference values or rule of thumb. Method 2 requires a population to be studied to characterise the statistical model, and method 3 requires a representative reference study. Given these limitations, method 1 seems preferable for correcting PWV per se for its blood pressure dependence. In summary, several options are available to handle the blood pressure dependence of PWV. If a blood pressure-independent index is sought, $\mathrm{CAVI}_{0}$ is theoretically preferable over CAVI. If correcting PWV per se is required, using an exponential pressure-area relationship provides the user with a method to correct PWV on an individual basis.

(c) 2017 S. Karger AG, Basel
\end{abstract}




\section{Arterial Stiffness}

The Recommendations for Improving and Standardizing Vascular Research on Arterial Stiffness by the American Heart Association have formulated several future needs in the field of arterial stiffness [1]. One of these needs is the development of therapeutic interventions to reduce arterial stiffness. Two potential interventions to reduce arterial stiffness are currently available: (1) blood pressure-lowering drugs, acting indirectly on the arterial wall by reducing cyclical pressure load, and (2) interventions that act directly on the arterial wall.

When assessing interventions of the first category, care should be taken since blood pressure is an intrinsic determinant of (carotid-femoral) pulse wave velocity (PWV), the gold standard for assessing arterial stiffness [2-4]. Therefore, the design as well as the outcome of studies investigating the effect of blood pressure-lowering drugs on the arterial wall require careful consideration of the intrinsic dependence of PWV on blood pressure. This emphasises that independent quantification of arterial stiffness is not trivial, and that a change in measured PWV cannot be directly and unambiguously interpreted as a change in intrinsic arterial wall stiffness. In particular, the intrinsic material stiffness of the arterial wall (i.e., the incremental elastic modulus) itself depends on pressure [5]. We recently set out to quantitatively assess the physiological confounders of PWV [1], investigating the magnitude of their effect and ways to correct for them. These confounders included blood pressure [6-8], heart rate $[9,10]$, and axial stretch [11]. This mini-review will concentrate on blood pressure as a confounder of PWV, and will discuss several options to deal with the confounding effect. Arterial stiffness measures that are not PWV-based (e.g., ambulatory arterial stiffness index [12], augmentation index [13]) or those based on the comparison of multiple PWVs at different sites (e.g., PWV ratio [14-16]) are outside the scope of this mini-review.

\section{Options for Handling the Pressure Dependence of Arterial Stiffness}

Current recommendations by the American Heart Association [1] emphasise blood pressure as a confounder of PWV. PWV has long been known to change with blood pressure $[2,3]$. Because of this dependence, PWV must be corrected for blood pressure if one wishes to assess arterial stiffness independently. There are two main options to accomplish this: (1) by converting PWV into another dimensionless measure that is blood pressure-independent, or (2) by correcting for the pressure dependence of PWV, but keeping PWV as the output measure, which is still in $\mathrm{m} / \mathrm{s}$.

This mini-review will first focus on the conversion of PWV into blood pressure-independent indices (option 1). Subsequently, correction of PWV will be briefly discussed (option 2).

\section{Option 1: Converting PWV into Blood Pressure-Independent Measures: Cardio-Ankle Vascular Index (CAVI) and $\mathrm{CAVI}_{0}$}

In 2006, Shirai et al. [17] proposed CAVI. CAVI is a measure of the stiffness of the heartto-ankle vascular bed, and is based on the PWV along this trajectory. Estimated PWV is converted into CAVI as follows:

$$
\mathrm{CAVI}=\ln \left(\frac{P_{\mathrm{s}}}{P_{\mathrm{d}}}\right) \times \frac{\mathrm{PWV}^{2} \times 2 \rho}{P_{\mathrm{s}}-P_{\mathrm{d}}},
$$


Spronck et al.: Options for Dealing with Pressure Dependence of Pulse Wave Velocity as a Measure of Arterial Stiffness: An Update of CAVI and CAVI

where $P_{\mathrm{s}}$ and $P_{\mathrm{d}}$ are systolic and diastolic blood pressures, and $\rho$ is the blood mass density $[17,18]$. Pressures, $\mathrm{PWV}$, and $\rho$ should be entered into equation 1 in units of $\mathrm{Pa}, \mathrm{m} / \mathrm{s}$, and $\mathrm{kg} /$ $\mathrm{m}^{3}$, respectively to ensure dimensional correctness.

Fukuda Denshi (Tokyo, Japan), the company that has patented CAVI, also produces a device ("VaSera") that can directly measure and calculate CAVI in humans. CAVI as output by the VaSera device $\left(\mathrm{CAVI}_{\mathrm{VS}}\right)$ is related to $\mathrm{CAVI}$ from equation 1 as follows: $\mathrm{CAVI}_{\mathrm{VS}}=a \times \mathrm{CAVI}+$ $b$, where $a$ and $b$ are proprietary constants [17].

CAVI is based on the observation by Hayashi et al. [19] that blood pressure and arterial diameter relate exponentially. The exponential pressure-diameter relation, when combined with the Bramwell-Hill equation that relates PWV to changes in blood pressure and diameter [20], yields equation 1 . Although named "cardio-ankle," the CAVI formula can equally well be applied to any PWV measurement in pulsatile pressure vessels, including the carotid-femoral PWV [21]. For a comprehensive overview of CAVI literature, the reader is referred to the reviews by Asmar [22], Shirai et al. [23, 24], Saiki et al. [25], and Miyoshi and Ito [26].

\section{Dependence of CAVI on Blood Pressure: Statistical Observations}

CAVI is claimed to be a blood pressure-independent measure. Several studies have investigated CAVI's dependence on blood pressure [17, 27-32]. Some of these studies assessed the blood pressure dependence cross-sectionally, with some showing a significant blood pressure dependence of CAVI [27] and others not showing a dependence [17]. As indicated by Shirai et al. [23], results from such cross-sectional studies do not necessarily translate to CAVI's blood pressure dependence at the time of measurement. In fact, a cross-sectional correlation between CAVI and blood pressure may be caused by hypertensive patients actually having stiffer arteries, and not by an intrinsic blood pressure dependence [33].

Subsequent studies have investigated CAVI's blood pressure dependence longitudinally $[28,29,31,32]$. Shirai et al. [28] studied the response of CAVI to two blood pressurelowering agents: metoprolol, a $\beta_{1}$ receptor blocker, and doxazosin, an $\alpha_{1}$ receptor blocker. Metoprolol, decreasing blood pressure through decreasing cardiac output, is thought not to have much effect on vascular tone, whereas doxazosin will cause vasodilatation. Shirai et al. [28] found that CAVI did not change with blood pressure reduction by metoprolol, but did change with doxazosin. From this study, they concluded that CAVI is blood pressure-independent, a conclusion that is recapitulated in Shirai et al. [29]. However, a limitation of this study appears to be that it was performed in only 12 subjects. Therefore, the observation that metoprolol did not change CAVI cannot be interpreted as a proof that CAVI does not change with blood pressure, because such a study may lack statistical power [30]. ${ }^{1}$ In 2015 , Lim et al. [31] assessed variation of CAVI during various blood pressure interventions (head-up/headdown tilt, mental stress, isometric handgrip exercise, cold pressor test). They found a substantial dependence of CAVI on mean blood pressure, with blood pressure changed using an isometric handgrip exercise (after heart rate correction), as well as an overall heart rate

1 Shirai et al. [28] observed a metoprolol-induced blood pressure decrease from $\mathrm{t}=0 \mathrm{~h}$ to $\mathrm{t}=3 \mathrm{~h}$ from $131.4 / 85.3$ to $118.3 / 75.3 \mathrm{~mm} \mathrm{Hg}$ (systolic/diastolic blood pressure; both $p<0.05$ ). At $\mathrm{t}=0 \mathrm{~h}$, CAVI was 8.16. Based on theoretical blood pressure dependence only [7], at $\mathrm{t}=3 \mathrm{~h}$, CAVI would change to 8.00 at $\mathrm{t}=3 \mathrm{~h}, \mathrm{a}$ decrease of 0.16 . The standard deviation of repetitive CAVI measurements in a single individual has been reported to be 0.28 [17], which translates to a standard deviation of the difference between two measurements of $0.28 \cdot \sqrt{2}=0.40$. Given these numbers, paired $t$ test sample size calculation $(\alpha=0.05,1-\beta=0.80)$ yields a required number of subjects of 52 to show a statistically significant change in CAVI with the observed metoprolol-induced blood pressure changes. At the actual sample size used $(n=12)$ [28], the power $(1-\beta)$ to detect CAVI's theoretical blood pressure dependence, given the observed blood pressure change, was 0.24. 
Spronck et al:: Options for Dealing with Pressure Dependence of Pulse Wave Velocity as a Measure of Arterial Stiffness: An Update of CAVI and CAVI $I_{0}$

dependence. However, this dependence may have been caused by a change in autonomic nervous activation caused by the handgrip exercise, which could have modulated the vascular tone of the cardio-ankle vascular bed. Ibata et al. [32] studied the short-term response of CAVI to physical exercise. With exercise and the corresponding increase in blood pressure, CAVI was found to increase significantly. Again, in this study, the observed blood pressure dependence may have been caused by a change in vascular tone due to autonomic nervous activation.

\section{Dependence of CAVI on Blood Pressure: Theoretical Underpinning}

The previous paragraph focussed on CAVI's blood pressure dependence from a statistical point of view. In a recent Journal of Hypertension publication, we studied CAVI's relationship to blood pressure from a theoretical standpoint [7]. We showed that, on a theoretical basis, the use of equation 1 to obtain CAVI does not yield a fully pressure-independent index. In short, the derivation of this equation results in a pressure dependence in two ways:

(1) CAVI is essentially a form of arterial stiffness index $\beta$. The derivation of stiffness index $\beta$ is based on findings by Hayashi et al. [19] that the relationship between arterial pressure $(P)$ and diameter $(d)$ is exponential:

$$
P=P_{\text {ref }} \times e^{\beta_{0}\left[\left(d / d_{\text {ret }}\right)-1\right]},
$$

where $P_{\text {ref }}$ and $d_{\text {ref }}$ indicate a reference pressure and the corresponding diameter. This equation, however, contains the index $\beta_{0}$, which is not equal to $\beta$. Stiffness index $\beta$ as commonly used clinically is defined as

$$
\beta=\frac{\ln \left(\frac{P_{\mathrm{s}}}{P_{\mathrm{d}}}\right)}{\frac{d_{\mathrm{s}}}{d_{\mathrm{d}}}-1},
$$

with $d_{\mathrm{s}}$ and $d_{\mathrm{d}}$ systolic and diastolic diameters, respectively. $\beta$ is obtained by substituting reference pressure and diameter $\left(P_{\text {ref }}\right.$ and $\left.d_{\text {ref }}\right)$ in equation 2 with diastolic pressure and diameter [34]. The resulting exponent $(\beta)$ is not equal to $\beta_{0}$. This causes what is supposed to be a fixed reference pressure $\left(P_{\text {ref }}\right)$ to vary with blood pressure. Consequently, $\beta$ also varies with blood pressure. As CAVI is essentially a form of $\beta$, this blood pressure dependence is also present in CAVI [7]. Segers [35] acknowledges this effect in his editorial comment, in which he illustrates the blood pressure dependence of $\beta$ by plotting $\operatorname{In}\left(P / P_{\text {ref }}\right)$ versus $d / d_{\text {ref. }}$. The slope of this plot, which is equal to $\beta$ [18], changes when changing $P_{\text {ref }}$ and $d_{\text {ref, }}$ as is the case when diastolic blood pressure is used in calculating $\beta$.

(2) The underlying Bramwell-Hill equation uses a coarse, linear approximation of the derivative of the arterial pressure-diameter relationship, whereas an exact derivative of pressure to diameter can be analytically derived from equation 2 , which can subsequently be evaluated in the diastolic point.

In a simulation study $(n=161)$, simulating the effects of blood pressure-lowering medication on CAVI, we showed that the pressure dependence of CAVI can lead to erroneous conclusions about an intrinsically de-stiffened arterial wall, even in controlled intervention trials. As emphasised by Segers [35], the order of magnitude of our simulated effect is equal to the differences found in some intervention studies that use CAVI as an arterial stiffness measure [36]. This indicates the importance of being aware of this theoretical blood pressure dependence, as CAVI is increasingly being used to assess blood pressure-independent effects on arterial stiffness [22-26]. 
Correcting the Theoretical Dependence of CAVI on Blood Pressure: CAVI ${ }_{0}$

To overcome the problem of CAVI's blood pressure dependence, we derived an updated index that is theoretically pressure-independent: $\mathrm{CAVI}_{0}$ [7]. $\mathrm{CAVI}_{0}$ is based on $\beta_{0}$, as opposed to CAVI that is based on (Kawasaki's) $\beta$ :

$$
\begin{aligned}
\beta_{0}= & \beta-\ln \left(\frac{P_{\mathrm{d}}}{P_{\text {ref }}}\right) \\
= & \frac{\ln \left(\frac{P_{\mathrm{s}}}{P_{\mathrm{d}}}\right)}{\frac{d_{\mathrm{s}}}{d_{\mathrm{d}}}-1}-\ln \left(\frac{P_{\mathrm{d}}}{P_{\text {ref }}}\right) .
\end{aligned}
$$

As $C A V I_{0}$ is based on $\beta_{0}, \mathrm{CAVI}_{0}$ does not show the "varying reference pressure-induced" blood pressure dependence that CAVI shows. Furthermore, we used the exact diastolic derivative of the pressure-diameter relationship which is consistent with using PWV, i.e., wave velocity at diastolic pressure, as a measured input. Taken together, this yielded the following equation for $\mathrm{CAVI}_{0}$ :

$$
\mathrm{CAVI}_{0}=\frac{\mathrm{PWV}^{2} \times 2 \rho}{P_{\mathrm{d}}}-\ln \left(\frac{P_{\mathrm{d}}}{P_{\text {ref }}}\right) .
$$

Pressures, PWV, and $\rho$ should be entered into this equation in units of $\mathrm{Pa}, \mathrm{m} / \mathrm{s}$, and $\mathrm{kg} / \mathrm{m}^{3}$, respectively to ensure dimensional correctness. The use of $\mathrm{CAVI}_{0}$ and $\beta_{0}$ requires the use of a reference pressure $\left(P_{\text {ref }}\right)$. It is important that one, fixed value for $P_{\text {ref }}$ is chosen for all subjects in a study. The numerical value of $P_{\text {ref }}$ that is chosen is a matter of standardisation or consensus; $P_{\text {ref }}$ does not represent a physiological pressure [7]. Following Hayashi et al. [19], we proposed to use $P_{\text {ref }}=100 \mathrm{~mm} \mathrm{Hg}$ in all subjects, in all studies. This ensures maximum comparability between $\beta_{0}$ and/or $\mathrm{CAVI}_{0}$ values among studies.

In the aforementioned simulation study, we also tested $\mathrm{CAVI}_{0}$, and found no blood pressure dependence of $\mathrm{CAVI}_{0}$ [7]. We would like to emphasise that the derivation and simulation of $\mathrm{CAVI}_{0}$ were performed under the assumption that the relationship between arterial pressure and diameter is truly exponential, an assumption that was also used by the developers of the original CAVI [17]. In summary, $\mathrm{CAVI}_{0}$ is an index of similar complexity as CAVI, but with less simplifying assumptions to be made for its derivation. In this light, we propose that $\mathrm{CAVI}_{0}$ is preferable over CAVI. The proposal of $\mathrm{CAVI}_{0}$ is relatively recent at publication of this mini-review, and it remains for population studies and retrospective application of the index to be studied to quantify the degree to which the blood pressure independence as seen in terms of theory has a practical advantage over CAVI.

\section{Option 2: Correcting PWV per se}

As mentioned previously, PWV per se can also be corrected for blood pressure. This can be accomplished in several ways, including: (1) correcting PWV based on a blood pressure dependence derived from an exponential relationship between pressure and cross-sectional area; (2) correcting PWV for blood pressure on a statistical basis; and (3) using reference values or a rule of thumb to correct PWV.

Method 1 gives direct insight into the magnitude of the pressure dependence of PWV. We used this method to correct local carotid PWVs in a population of hypertensives that were measured before and after hypertensive treatment [6]. In this study, we found that the predicted change in PWV due to blood pressure lowering (assuming an exponential pressurearea relationship) was of equal magnitude as the measured change. This suggests that during 
the follow-up period in this study (3 months), no arterial remodelling took place in the artery assessed using PWV (the carotid artery in this case). We further assessed the performance of this method in a data set of subjects that were treated using antiangiogenic drugs [8]. In the same study, we also compared method 1 to a purely statistical approach (method 2). The numerical results of these approaches were similar. Although commonly used in research studies, a statistical approach is only applicable if a population or a group of subjects is studied, i.e., it is not applicable in individuals. A possible solution to this problem is to relate a PWV measurement to a blood pressure-specific reference value, e.g., from the Reference Values for Arterial Stiffness' Collaboration 2010 study [37] (method 3). Nonetheless, the values in this reference values study represent both acute and (long-term) remodelling effects of blood pressure on PWV [33]. Therefore, the reference values from the Reference Values for Arterial Stiffness' Collaboration 2010 do not independently quantify the physiological confounding effect of blood pressure on PWV.

Theoretically, a generic, population-based statistical model could be constructed of the intrinsic pressure dependence of $\mathrm{PWV}$, as a function of age and other potential confounders such as diabetic status. Construction of such a model would require measurements of the acute pressure dependence of PWV (e.g., by manipulating blood pressure through metoprolol). Notably, the resulting statistical model would differ from the model as presented for example by the Reference Values for Arterial Stiffness' Collaboration [37]. As discussed in the previous paragraph, in the latter study, no distinction was made between acute and (longterm) remodelling effects of blood pressure on PWV, resulting in a statistical model that describes both these effects.

A very simple type of "reference value" is a rule of thumb. As presented in Spronck et al. [6], PWV changes by approximately $1 \mathrm{~m} / \mathrm{s}$ per $10 \mathrm{~mm} \mathrm{Hg}$ change in diastolic blood pressure. Using such a rule of thumb has the clear advantage of being easily applicable in the clinic. However, the mentioned paper also shows that the actual pressure dependence of PWV is age-specific [6].

In our analysis [6], a change in blood pressure from 120/80 to $160 / 90 \mathrm{~mm} \mathrm{Hg}$ leads to a change in PWV of $0.9 \mathrm{~m} / \mathrm{s}$ in subjects with a mean age of 41 years, but to a change of $1.3 \mathrm{~m} / \mathrm{s}$ in subjects with a mean age of 64 years. These numbers are slightly lower than those calculated from the Reference Values for Arterial Stiffness' Collaboration 2010 data [37]. This difference might be caused by the remodelling effect of high blood pressure on arterial stiffness.

Given the limitations of methods 2 and 3, method 1 seems preferable for calculating the acute effect of blood pressure on PWV.

Which Blood Pressure Component to Use for Statistical Correction of PWV?

When correcting for blood pressure statistically (method 2), one has to choose which blood pressure component (diastolic, mean, systolic blood pressure, or pulse pressure) to use for correction. Current recommendations state that mean arterial pressure should be taken into consideration when PWV data are analysed [1].

Several studies [38-40] assessed the relationship between PWV and these blood pressure components cross-sectionally. They found a significant correlation of PWV with all pressure component measures except diastolic blood pressure. Sa Cunha et al. [41] report a correlation between PWV and systolic blood pressure for both genders, and a correlation between PWV and diastolic blood pressure only in women. In contrast, Nürnberger et al. [42] only report a correlation between PWV and diastolic blood pressure. What all these studies have in common is that they assessed the association between PWV and blood pressure cross-sectionally. As explained previously in the context of CAVI, the relationship that is obtained in this way may not reflect the acute effect of blood pressure on PWV. 
Spronck et al.: Options for Dealing with Pressure Dependence of Pulse Wave Velocity as a Measure of Arterial Stiffness: An Update of CAVI and CAVI

Theoretically and empirically, it can be shown that PWV varies with diastolic blood pressure $[2,3,43,44]$, since the velocity measurement is conventionally made using the diastolic foot of the pressure wave as the fiducial reference point. Therefore, the propagating pulse wave in this context "sees" the diastolic pressure. In other words, the front of the pressure wave travelling to the periphery encounters an arterial section that is (still) at diastolic blood pressure.

As is clear from the above, the question of which blood pressure component to use for correction of PWV can be approached from (1) a cross-sectional, population perspective or (2) an acute, mechanistic perspective. If one wishes to correct a measurement set on a statistical basis for the influence of blood pressure, mean, systolic, and diastolic blood pressure are all good candidates, depending on the application and population subtype. If, on the other hand, one wants to remove the acute effect of blood pressure on a PWV measurement in an individual subject, (the subject's own) diastolic blood pressure is a well-founded choice.

\section{Summary}

Several options are available to handle the blood pressure dependence of PWV, all having their pros and cons. If a blood pressure-independent index is preferable, we propose that $\mathrm{CAVI}_{0}$ is, at least theoretically, preferable over CAVI in terms of pressure independence. Whether this theoretical benefit also translates to the clinical practice remains to be confirmed. If correcting PWV per se is required, using an exponential pressure-area relationship provides the user with a method to correct PWV on an individual basis.

\section{Acknowledgement}

This study was supported by an Endeavour Research Fellowship awarded by the Australian Government to B. Spronck.

\section{Disclosure Statement}

The authors have no conflicts of interest to disclose.

\section{References}

1 Townsend RR, Wilkinson IB, Schiffrin EL, Avolio AP, Chirinos JA, Cockcroft JR, Heffernan KS, Lakatta EG, McEniery CM, Mitchell GF, Najjar SS, Nichols WW, Urbina EM, Weber T; American Heart Association Council on Hypertension: Recommendations for Improving and Standardizing Vascular Research on Arterial Stiffness: a scientific statement from the American Heart Association. Hypertension 2015;66:698-722.

2 Bramwell JC, McDowall RJS, McSwiney BA: The variation of arterial elasticity with blood pressure in man (part I). R Soc Lond Proc B 1923;94:450-454.

-3 Nye ER: The effect of blood pressure alteration on the pulse wave velocity. Br Heart J 1964;26:261-265.

-4 Laurent S, Cockcroft J, Van Bortel L, Boutouyrie P, Giannattasio C, Hayoz D, Pannier B, Vlachopoulos C, Wilkinson I, Struijker-Boudier H; European Network for Non-invasive Investigation of Large Arteries: Expert consensus document on arterial stiffness: methodological issues and clinical applications. Eur Heart J 2006; 27:2588-2605.

5 Bergel DH: The static elastic properties of the arterial wall. J Physiol 1961;156:445-457.

-6 Spronck B, Heusinkveld MH, Vanmolkot FH, Op 't Roodt J, Hermeling E, Delhaas T, Kroon AA, Reesink KD: Pressure-dependence of arterial stiffness: potential clinical implications. J Hypertens 2015;33:330-338.

$\checkmark 7$ Spronck B, Avolio AP, Tan I, Butlin M, Reesink KD, Delhaas T: Arterial stiffness index beta and cardio-ankle vascular index inherently depend on blood pressure but can be readily corrected. J Hypertens 2017;35: 98-104. 
Spronck et al.: Options for Dealing with Pressure Dependence of Pulse Wave Velocity as a Measure of Arterial Stiffness: An Update of CAVI and CAVI

-8 Spronck B, Delhaas T, De Lepper AG, Giroux J, Goldwasser F, Boutouyrie P, Alivon M, Reesink KD: Patientspecific blood pressure correction technique for arterial stiffness: evaluation in a cohort on anti-angiogenic medication. Hypertens Res 2017;40:752-757.

-9 Tan I, Spronck B, Kiat H, Barin E, Reesink KD, Delhaas T, Avolio AP, Butlin M: Heart rate dependency of large artery stiffness. Hypertension 2016;68:236-242.

10 Spronck B, Delhaas T, Op 't Roodt J, Reesink KD: Carotid artery applanation tonometry does not cause significant baroreceptor activation. Am J Hypertens 2016;29:299-302.

$>11$ Holtackers RJ, Spronck B, Heusinkveld MHG, Crombag G, Op 't Roodt J, Delhaas T, Kooi ME, Reesink KD, Hermeling E: Head orientation should be considered in ultrasound studies on carotid artery distensibility. J Hypertens 2016;34:1551-1555.

-12 Li Y, Wang JG, Dolan E, Gao PJ, Guo HF, Nawrot T, Stanton AV, Zhu DL, O’Brien E, Staessen JA: Ambulatory arterial stiffness index derived from 24-hour ambulatory blood pressure monitoring. Hypertension 2006;47: 359-364.

13 O'Rourke MF, Kelly RP: Wave reflection in the systemic circulation and its implications in ventricular function. J Hypertens 1993;11:327-337.

14 Fortier C, Sidibe A, Desjardins MP, Marquis K, De Serres SA, Mac-Way F, Agharazii M: Aortic-brachial pulse wave velocity ratio: a blood pressure-independent index of vascular aging. Hypertension 2017;69:96-101.

15 Fortier C, Mac-Way F, Desmeules S, Marquis K, De Serres SA, Lebel M, Boutouyrie P, Agharazii M: Aorticbrachial stiffness mismatch and mortality in dialysis population. Hypertension 2015;65:378-384.

16 Fortier C, Agharazii M: Arterial stiffness gradient. Pulse (Basel) 2016;3:159-166.

17 Shirai K, Utino J, Otsuka K, Takata M: A novel blood pressure-independent arterial wall stiffness parameter; cardio-ankle vascular index (CAVI). J Atheroscler Thromb 2006;13:101-107.

18 Hayashi K, Yamamoto T, Takahara A, Shirai K: Clinical assessment of arterial stiffness with cardio-ankle vascular index: theory and applications. J Hypertens 2015;33:1742-1757.

19 Hayashi K, Handa H, Nagasawa S, Okumura A, Moritake K: Stiffness and elastic behavior of human intracranial and extracranial arteries. J Biomech 1980;13:175-184.

20 Bramwell JC, Hill AV: The velocity of the pulse wave in man. R Soc Lond Proc B 1922;93:298-306.

$\checkmark 21$ Wohlfahrt P, Krajcoviechova A, Seidlerova J, Mayer O, Bruthans J, Filipovsky J, Laurent S, Cifkova R: Arterial stiffness parameters: how do they differ? Atherosclerosis 2013;231:359-364.

22 Asmar R: Principles and usefulness of the cardio-ankle vascular index (CAVI): a new global arterial stiffness index. Eur Heart J Suppl 2017;19:B4-B10.

-23 Shirai K, Hiruta N, Song M, Kurosu T, Suzuki J, Tomaru T, Miyashita Y, Saiki A, Takahashi M, Suzuki K, Takata M: Cardio-ankle vascular index (CAVI) as a novel indicator of arterial stiffness: theory, evidence and perspectives. J Atheroscler Thromb 2011;18:924-938.

24 Shirai K, Saiki A, Nagayama D, Tatsuno I, Shimizu K, Takahashi M: The role of monitoring arterial stiffness with cardio-ankle vascular index in the control of lifestyle-related diseases. Pulse (Basel) 2015;3:118-133.

25 Saiki A, Sato Y, Watanabe R, Watanabe Y, Imamura H, Yamaguchi T, Ban N, Kawana H, Nagumo A, Nagayama D, Ohira M, Endo K, Tatsuno I: The role of a novel arterial stiffness parameter, cardio-ankle vascular index (CAVI), as a surrogate marker for cardiovascular diseases. J Atheroscler Thromb 2016;23:155-168.

26 Miyoshi T, Ito H: Assessment of arterial stiffness using the cardio-ankle vascular index. Pulse (Basel) 2016;4: $11-23$.

27 Kubozono T, Miyata M, Ueyama K, Nagaki A, Otsuji Y, Kusano K, Kubozono O, Tei C: Clinical significance and reproducibility of new arterial distensibility index. Circ J 2007;71:89-94.

28 Shirai K, Song M, Suzuki J, Kurosu T, Oyama T, Nagayama D, Miyashita Y, Yamamura S, Takahashi M: Contradictory effects of $\beta 1$ - and $\alpha 1$-adrenergic receptor blockers on cardio-ankle vascular stiffness index (CAVI) CAVI independent of blood pressure. J Atheroscler Thromb 2011;18:49-55.

29 Shirai K, Shimizu K, Takata M, Suzuki K: Independency of the cardio-ankle vascular index from blood pressure at the time of measurement. J Hypertens 2017;35:1521-1523.

30 Spronck B, Avolio AP, Tan I, Butlin M, Reesink KD, Delhaas T: Reply: physics cannot be disputed. J Hypertens 2017;35:1523-1525.

31 Lim J, Pearman ME, Park W, Alkatan M, Machin DR, Tanaka H: Impact of blood pressure perturbations on arterial stiffness. Am J Physiol Regul Integr Comp Physiol 2015;309:R1540-R1545.

-32 Ibata J, Sasaki H, Kakimoto T, Matsuno S, Nakatani M, Kobayashi M, Tatsumi K, Nakano Y, Wakasaki H, Furuta H, Nishi M, Nanjo K: Cardio-ankle vascular index measures arterial wall stiffness independent of blood pressure. Diabetes Res Clin Pract 2008;80:265-270.

33 Benetos A: Assessment of arterial stiffness in an older population: the interest of the cardio-ankle vascular index (CAVI). Eur Heart J Suppl 2017;19:B11-B16.

-34 Kawasaki T, Sasayama S, Yagi S, Asakawa T, Hirai T: Non-invasive assessment of the age related changes in stiffness of major branches of the human arteries. Cardiovasc Res 1987;21:678-687.

35 Segers P: A lesson in vigilance: pressure dependency of a presumed pressure-independent index of arterial stiffness. J Hypertens 2017;35:33-35.

-36 Kurata M, Okura T, Watanabe S, Irita J, Enomoto D, Johtoku M, Miyoshi K, Koresawa M, Fukuoka T, Higaki J: Effects of amlodipine and candesartan on arterial stiffness estimated by cardio-ankle vascular index in patients with essential hypertension: a 24-week study. Curr Ther Res Clin Exp 2008;69:412-422. 
-37 Reference Values for Arterial Stiffness' Collaboration: Determinants of pulse wave velocity in healthy people and in the presence of cardiovascular risk factors: "establishing normal and reference values." Eur Heart J 2010;31:2338-2350.

38 Kim EJ, Park CG, Park JS, Suh SY, Choi CU, Kim JW, Kim SH, Lim HE, Rha SW, Seo HS, Oh DJ: Relationship between blood pressure parameters and pulse wave velocity in normotensive and hypertensive subjects: invasive study. J Hum Hypertens 2007;21:141-148.

-39 Stompor T, Rajzer M, Sulowicz W, Dembinska-Kiec A, Janda K, Kawecka-Jaszcz K, Wojcik K, Tabor B, Zdzienicka A, Janusz-Grzybowska E: An association between aortic pulse wave velocity, blood pressure and chronic inflammation in ESRD patients on peritoneal dialysis. Int J Artif Organs 2003;26:188-195.

40 Tanaka H, DeSouza CA, Seals DR: Absence of age-related increase in central arterial stiffness in physically active women. Arterioscler Thromb Vasc Biol 1998;18:127-132.

-41 Sa Cunha R, Pannier B, Benetos A, Siché JP, London GM, Mallion JM, Safar ME: Association between high heart rate and high arterial rigidity in normotensive and hypertensive subjects. J Hypertens 1997;15:1423-1430.

-42 Nürnberger J, Dammer S, Saez AO, Philipp T, Schäfers RF: Diastolic blood pressure is an important determinant of augmentation index and pulse wave velocity in young, healthy males. J Hum Hypertens 2003;17:153-158.

43 Nichols WW, O’Rourke MF, Vlachopoulos C (eds): McDonald's Blood Flow in Arteries: Theoretical, Experimental and Clinical Principles. London, UK, CRC Press, 2011, p 73.

-44 Gao M, Cheng HM, Sung SH, Chen CH, Olivier NB, Mukkamala R: Estimation of pulse transit time as a function of blood pressure using a nonlinear arterial tube-load model. IEEE Trans Biomed Eng 2017;64:1524-1534. 